\title{
Hedgehog signalling in medulloblastoma, glioblastoma and neuroblastoma
}

\author{
MEHDI H. SHAHI, AIALA LORENTE and JAVIER S. CASTRESANA
}

\author{
Unidad de Biología de Tumores Cerebrales, Universidad de Navarra, 31008 Pamplona, Spain
}

Received August 1, 2007; Accepted November 13, 2007

\begin{abstract}
We investigated a role for Hedgehog signalling in glioblastoma, neuroblastoma and medulloblastoma by studying the transcription of PTCH, SMO, GLII and GLI3 in a total of 25 cell lines by standard RT-PCR and qRT-PCR, before and after 5-aza-2'-deoxycytidine and trichostatin A (TSA) treatments. Also 25 glioblastoma samples were tested by qRT-PCR. We also performed real-time methylated specific PCR (qMSP) of the $S M O$ promoter region in DNA from 80 tumor samples (40 glioblastomas and 40 neuroblastomas) and from the 25 cell lines. We detected SMO promoter methylation in more than half of the cell lines and tumor samples. $P T C H$ expression in cell lines was lower than in normal controls, just the opposite to GLII. SMO and GLI3 expression were high and fully correlated in glioblastoma and medulloblastoma, although partially in neuroblastoma. Our results support the existence of Hedgehog signalling in glioblastoma and medulloblastoma, and to a lesser extent, in neuroblastoma.
\end{abstract}

\section{Introduction}

Genes of the Bmi-1, Notch, Wnt and Sonic Hedgehog (Shh) pathways are crucial for development and may also be involved in stem cell regeneration. The Shh pathway regulates the embryonic development of both invertebrates and vertebrates, and contributes to the formation of different organs and tissues, including the neural tube. Shh is a secreted protein released from Purkinje cells, being responsible for the patterning, polarity and development of the cerebellum. It binds to target cells presenting the twelve transmembrane receptor Patched (Ptch), prompting cell growth and proliferation. Shh deregu-

Correspondence to: Dr Javier S. Castresana, Unidad de Biología de Tumores Cerebrales, Universidad de Navarra, Irunlarrea 1, 31008 Pamplona, Spain

E-mail: jscastresana@unav.es

Abbreviations: DMEM, Dulbecco's modified Eagle's medium; MSP, methylation specific PCR; PCR, polymerase chain reaction; PNET, primitive neuroectodermal tumor; qMSP, real-time methylation specific PCR; qRT-PCR, quantitative (real-time) reverse transcribed-PCR; RT-PCR, reverse transcribed-PCR; Shh, Sonic Hedgehog; TFR, transferrin receptor; TSA, trichostatin A

Key words: PTCH, SMO, GLI1, GLI3, methylation lated expression leads to developmental abnormalities and tumor formation as in the case of pancreatic, colorectal, breast and prostate cancer, basal cell carcinoma and brain tumors, including medulloblastoma (1). The first evidence for Shh roles in tumorigenesis came from a patient suffering from Gorlin's Syndrome, a kind of familial predisposition to medulloblastoma, rhabdomyosarcoma and basal cell carcinoma (2).

In the absence of Shh, Ptch inhibits the seven transmembrane receptor Smoothened (Smo) (3). Shh-Ptch binding relieves Smo, that further activates the downstream target Gli1, and Patch itself $(4,5)$. The GLI gene family was first identified in glioblastoma (6). GLI1, GLI2 and GLI3 share five highly conserved tandem $\mathrm{C} 2 \mathrm{H} 2$ zinc finger domains and a histidinecysteine linker sequence in between the zinc fingers. In humans, GLII has two isoforms, GLI2 has three alternatively spliced exons, and GLI3 has only one isoform. Gli1 acts as an activator, Gli2 as an activator or as a repressor depending upon its catalytic activity, and Gli3 as a repressor (7-11).

It has recently been reported that functional Smo is required for $G L I 3$ expression in colorectal carcinoma cell lines and that $S M O$ methylation leads to silence of GLI3 expression independently of the Shh pathway (12). In order to prove this in malignant brain tumors, we selected 25 cell lines (6 of medulloblastoma, 8 of glioblastoma, and 11 of neuroblastoma) and 80 tumor samples (40 glioblastomas and 40 neuroblastomas) to check for PTCH, GLII, SMO and GLI3 expression, together with $S M O$ methylation. The expression was checked by standard RT-PCR and qRT-PCR and compared with normal adult brain RNA as a positive control. After treating the cell lines with 5-aza-2'-deoxycytidine and TSA (trichostatin A), mRNA expression was newly checked. We also checked SMO promoter methylation by real-time melting curve (qMSP) analysis in both tumor samples and cell lines.

\section{Materials and methods}

Cell lines and tumor samples. Twenty-five cell lines (6 from medulloblastoma, 8 from glioblastoma and 11 from neuroblastoma), together with 80 tumor samples (40 glioblastomas and 40 neuroblastomas) were analyzed in this study. For a complete list of the cell lines, see Table III. Medulloblastoma and glioblastoma cells were maintained in RPMI-1640 medium (Gibco/Invitrogen) supplemented with $10 \%$ fetal calf serum, $1 \%$ penicillin and $0.1 \%$ amphocin. For medulloblastoma cells, $4 \%$ non-essential amino acids were added. Neuroblastoma cell lines were maintained in DMEM medium (Gibco/Invitrogen) 
Table I. Primers used for RT-PCR and MSP analyses.

\begin{tabular}{|c|c|c|c|c|}
\hline Gene & Forward primers $\left(5^{\prime}-3^{\prime}\right)$ & Reverse primers $\left(5^{\prime}-3{ }^{\prime}\right)$ & $\mathrm{T}^{\mathrm{a}}\left({ }^{\circ} \mathrm{C}\right)$ & bp \\
\hline GAPDH (s) & GAAGGTGAAGGTCGGAGTCAAC & CAGAGTTAAAAGCAGCCCTGGT & 62 & 70 \\
\hline GLI1 (s) & TGTATGTAAGCTCCCTGGCT & AGTATAGGCAGAGCTGATGC & 59.2 & 577 \\
\hline SMO (s) & ACGAGGACGTGGAGGGCTG & CGCACGGTATCGGTAGTTCT & 55 & 580 \\
\hline TFR $(s)$ & GTCAATGTCCCAAACGTCACCAGA & ATTTCGGGAATGCTGAGAAAACAGACAGA & 60 & 300 \\
\hline PTCH1 $(s, q)$ & CTTCGCTCTGGAGCAGATTT & CAGGACATTAGCACCTTCT & 55 & 354 \\
\hline GLI3 (s,q) & CAGCTCCACGACCACTGAA & TCCATGGCAAACACCGTCC & 53 & 324 \\
\hline GLI1 (q) & CAGTGTGGGGACAGAAGGA & CGGGGAGAAGAAAAGAGTGG & 57.4 & 132 \\
\hline $\operatorname{SMO}(q)$ & AAGGCTGCACGAATGAGGT & GGGTTCTGGCACTGGATG & 57 & 133 \\
\hline SMO-M & TTTTTTTATTTTTTCGTTTTTTCGT & CCGACTCCTTTATTACTCTAACTCG & 51 & 125 \\
\hline SMO-U & TTTTTTATTTTTTTGTTTTTTTGT & ААСТССТТТАТТАСТСТААСТСАСТ & 51 & 122 \\
\hline
\end{tabular}

Primers used for standard RT-PCR (s), qRT-PCR (q) or both (s,q). SMO-M and SMO-U were used as methylation primers (M) and unmethylation primers (U) for qMSP. Ta, annealing temperature; bp, base pairs of the amplified product.

Table II. All techniques applied to explore PTCH, SMO, GLII and GLI3 in each group of cell lines and in the tumor samples.

\begin{tabular}{|c|c|c|c|c|}
\hline & PTCH & SMO & GLI1 & GLI3 \\
\hline $\begin{array}{l}\text { MB cell lines } \\
(6)\end{array}$ & RT-PCR ${ }^{\mathrm{a}}, \mathrm{qRT}^{\mathrm{P}} \mathrm{PCR}^{\mathrm{a}}$ & $\begin{array}{c}\text { RT-PCR }{ }^{\mathrm{a}}, \mathrm{qRT}^{\mathrm{T}}-\mathrm{PCR}^{\mathrm{a}} \\
\text { qMSP }\end{array}$ & RT-PCR, qRT-PCR & RT-PCR ${ }^{\mathrm{a}}, \mathrm{qRT}-\mathrm{PCR}^{\mathrm{a}}$ \\
\hline $\begin{array}{l}\text { NB cell lines } \\
(11)\end{array}$ & RT-PCR ${ }^{\mathrm{a}}, \mathrm{qRT}^{-\mathrm{PCR}^{\mathrm{a}}}$ & $\begin{array}{c}\text { RT-PCR }{ }^{\mathrm{a}}, \mathrm{qRT}^{\mathrm{R}}-\mathrm{PCR}^{\mathrm{a}} \\
\text { qMSP }\end{array}$ & RT-PCR, qRT-PCR & RT-PCR ${ }^{\mathrm{a}}$, qRT-PCR ${ }^{\mathrm{a}}$ \\
\hline $\begin{array}{l}\text { GB cell lines } \\
(8)\end{array}$ & RT-PCR ${ }^{\mathrm{a}}, \mathrm{qRT}^{-\mathrm{PCR}^{\mathrm{a}}}$ & $\begin{array}{c}\text { RT-PCR }^{\mathrm{a}}, \mathrm{qRT}^{-\mathrm{PCR}^{\mathrm{a}}} \\
\text { qMSP }\end{array}$ & RT-PCR, qRT-PCR & RT-PCR ${ }^{\mathrm{a}}$, qRT-PCR ${ }^{\mathrm{a}}$ \\
\hline GB samples (40) & qRT-PCR (25 samples) & $\begin{array}{c}\text { qRT-PCR (25 samples) } \\
\text { qMSP (40 samples) }\end{array}$ & qRT-PCR (25 samples) & qRT-PCR (25 samples) \\
\hline NB samples (40) & nd & qMSP (40 samples) & nd & nd \\
\hline
\end{tabular}

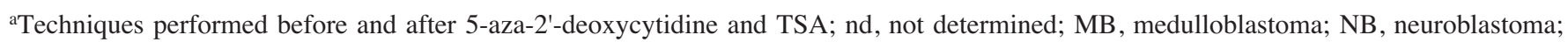
$\mathrm{GB}$, glioblastoma.

supplemented with $10 \%$ fetal calf serum, $5 \%$ non-essential amino acids, $1 \%$ penicillin and $0.1 \%$ amphocin.

Standard and quantitative RT-PCR. RNA was extracted from all cell line pellets, using the Quick Prep total-RNA extraction Kit (Amersham Biosciences, UK). Normal adult brain RNA (Stratagene, Cedar Creek, TX) and lung RNA (Chemicon International Inc., Temecula, CA), were used as positive controls of expression. RNA was converted to cDNA by the Superscript II RNase H Reverse Transcriptase kit (Invitrogen Life Technologies, Carlsbad, CA). The cDNAs were finally amplified by standard PCR by using the oligonucleotides in Table I. We also checked these gene expression levels in all the 25 cell lines and 25 glioblastoma samples by qRT-PCR. A complete list of techniques applied to explore $\mathrm{PTCH}$, $S M O, G L I 1$ and GLI3 in each group of cell lines and in the tumor samples is given in Table II. 5-aza-2'-deoxycytidine and TSA treatments of cells. Treatments of cells in culture were started when the confluence was about $30 \%\left(2.6 \times 10^{5}\right.$ cells $\left./ \mathrm{ml}\right)$. Five micrograms of 5-aza-2'deoxycytidine (Sigma-Aldrich) was used for $72 \mathrm{~h}$, and $100 \mathrm{ng} /$ $\mathrm{ml}$ TSA (Sigma-Aldrich) was applied for the next $24 \mathrm{~h}$. Cell media were changed every $24 \mathrm{~h}$. RNA extraction was performed before and after treatments with 5-aza-2'deoxycytidine and TSA, in order to perform cDNA synthesis, RT-PCR and qRT-PCR of PTCH, SMO and GLI3 and to compare their expression levels in the two conditions.

Bisulphite modification, amplification and melting curve analysis. We selected the SMO promoter region for MSP analysis (13) from a CG rich region spanning -753 to -303 bp 5 ' upstream of the SMO transcriptional start site. Bisulfite modification of genomic DNA extracted from the 25 cell lines and 80 tumor samples was applied by using the CpGenome 


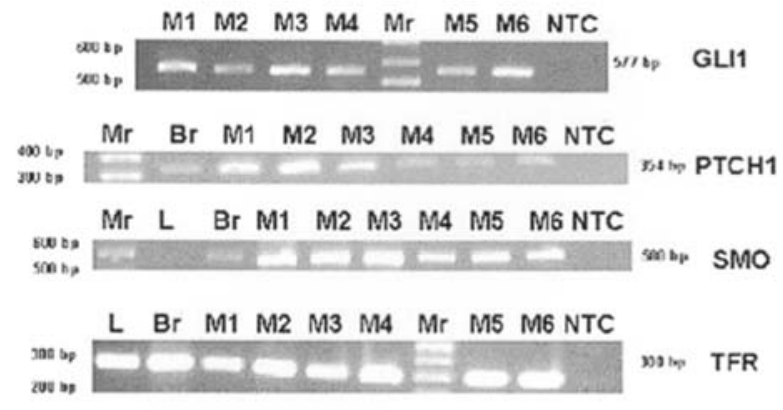

A

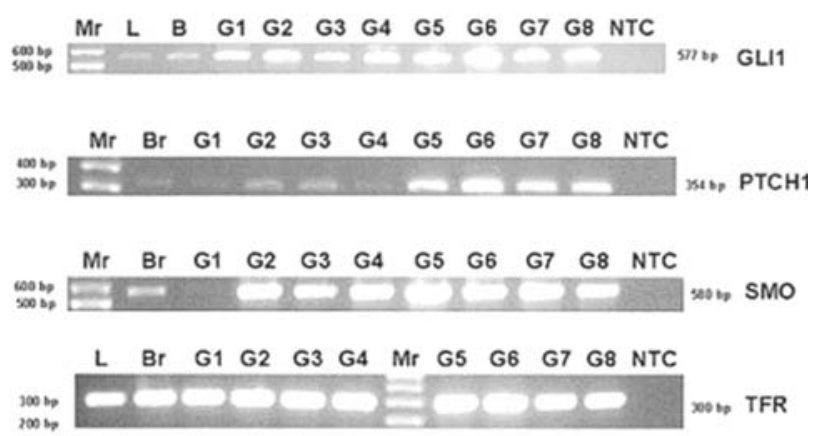

B

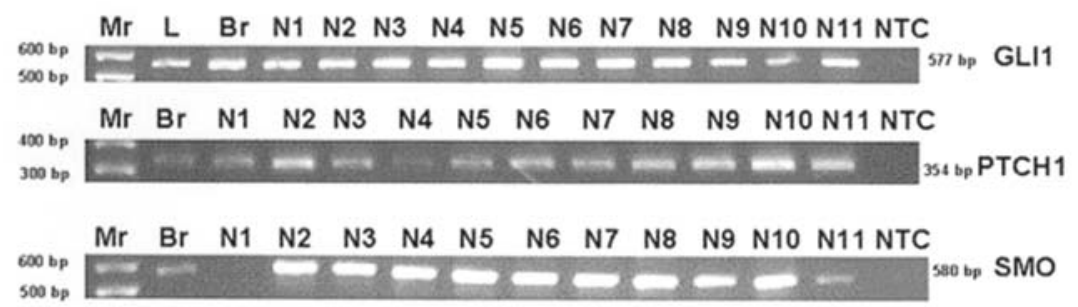

$\begin{array}{llllllllllllll}\mathrm{L} & \mathrm{Br} & \mathrm{N} 1 & \mathrm{~N} 2 & \mathrm{~N} 3 & \mathrm{~N} 4 & \mathrm{n} 5 & \mathrm{~N} 6 & \mathrm{~N} 7 & \mathrm{~N} 8 & \mathrm{~N} 9 & \mathrm{~N} 10 & \mathrm{~N} 11 & \mathrm{NTC}\end{array}$
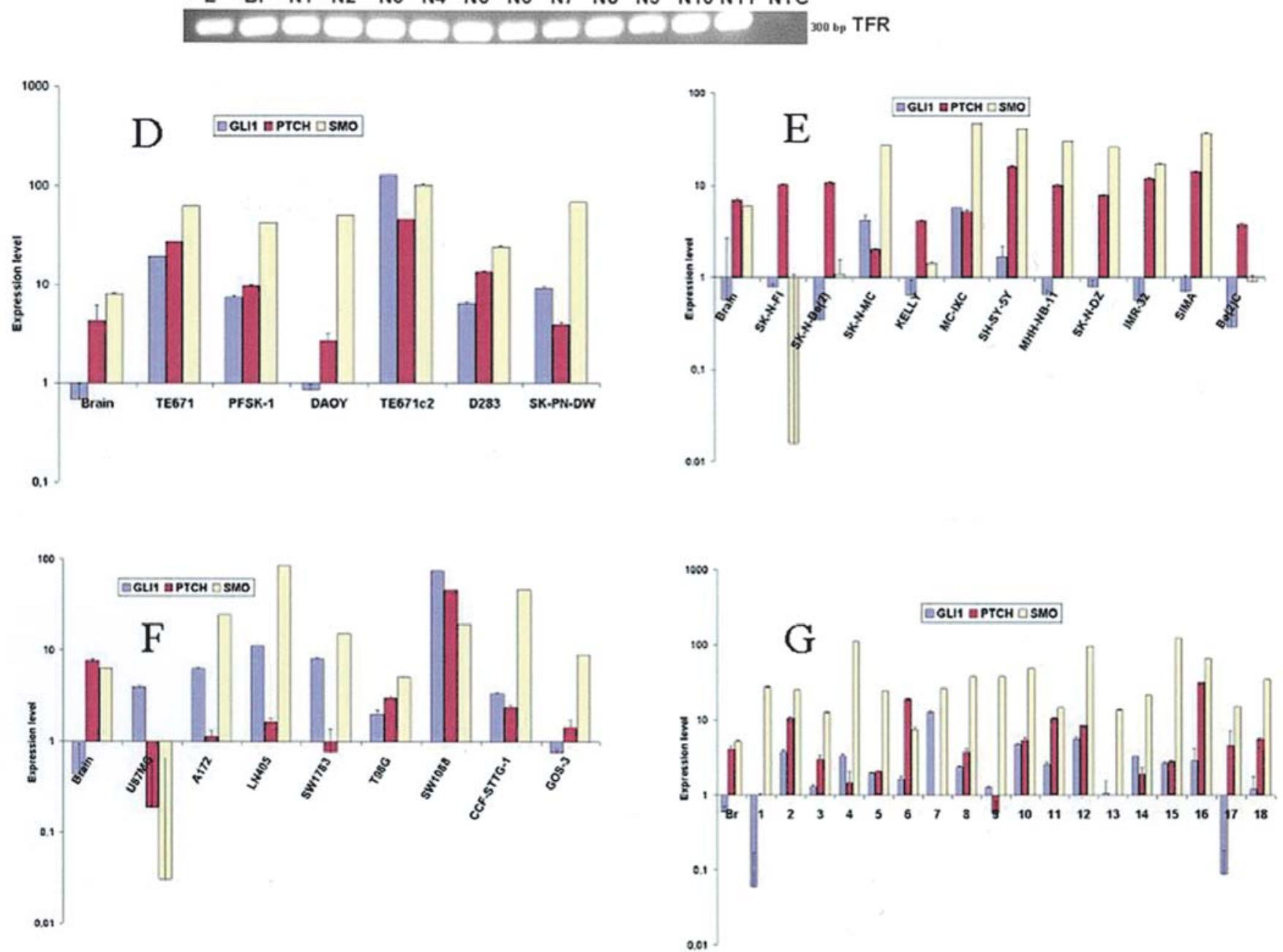

Figure 1. Comparative RNA expression of PTCH, SMO and GLII determined by standard (A-C) and quantitative (D-G) RT-PCR. M, medulloblastoma; N, neuroblastoma; G, glioblastoma; L, lung RNA; Br, normal adult brain RNA; NTC, non-template control; Mr, 1 Kb Plus DNA ladder (Invitrogen Life Technologies).

DNA modification Kit S7820 (Chemicon International). Two primer pairs were designed with the help of the online soft- ware Methprimer (http://www.urogene.org/methprimer/index1. html), and used for quantitative methylation-specific PCR 

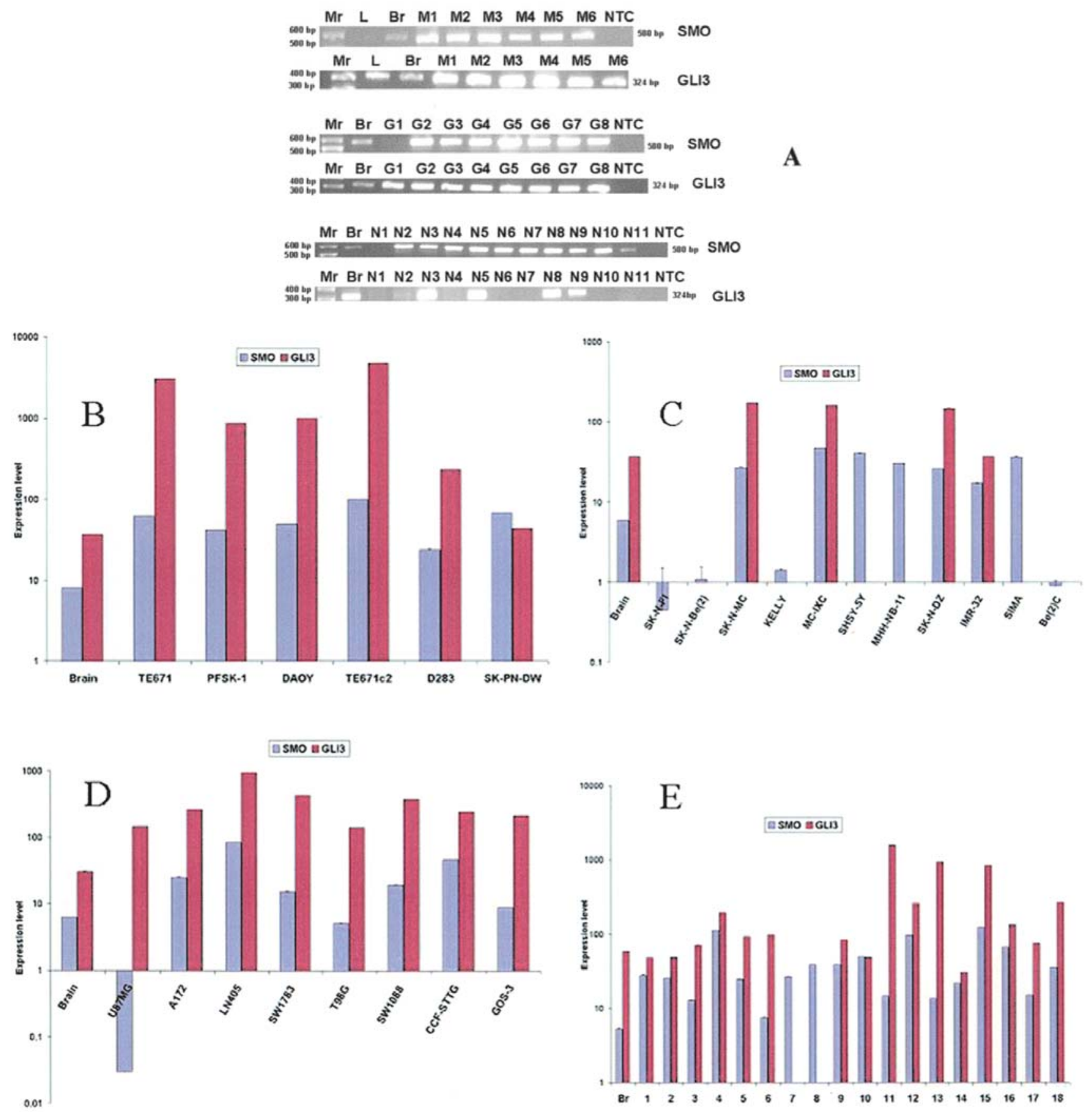

Figure 2. Comparative RNA expression of $S M O$ and GLI3 determined by standard (A) and quantitative (B-E) RT-PCR. M, medulloblastoma; N, neuroblastoma; G, glioblastoma; L, lung RNA; Br, normal adult brain RNA; NTC, non-template control; Mr, 1 Kb Plus DNA ladder (Invitrogen Life Technologies).

(qMSP) in an iQ 5 multicolor real-time PCR detection system (Bio-Rad). SYBER Green supermix (Bio-Rad) was used as PCR reagent. Each sample was tested in triplicate. Universal methylated DNA (Chemicon International) was included as a positive control, blood DNA as a negative control, and water (B. Braun Medical S.A., Barcelona) as a non-template control. The results were analyzed with the help of the melting curves produced by the methylated and unmethylated primers.

\section{Results}

PTCH, GLI1, SMO and GLI3 expression. All 6 medulloblastoma cell lines, 7 of 8 glioblastoma cell lines (except for U87MG), and 4 of 11 neuroblastoma cell lines (SK-N-MC,
MC-IXC, SK-N-DZ and IMR-32) expressed both SMO and GLI3 genes (Figs. 1 and 2, Table III). The rest of the neuroblastoma cell lines ( 7 of 11 ) expressed only SMO. The glioblastoma cell line U87MG only expressed GLI3. Most of the medulloblastoma, glioblastoma and neuroblastoma cell lines showed lower expression of PTCH than normal adult brain RNA controls (Fig. 1). After 5-aza-2'-deoxycytidine and TSA treatments PTCH expression was increased (Fig. 3, Table III). All 25 cell lines and 25 glioma samples expressed GLII at substantial quantity in comparison to normal controls (Fig. 1).

SMO promoter methylation. Four medulloblastoma cell lines (PFSK-1, TE671c2, D283 and SK-PN-DW) presented an increase in expression of the SMO gene after 5-aza-2'- 
Table III. PTCH, SMO and GLI3 expression in medulloblastoma, glioblastoma and neuroblastoma cell lines, before and after 5'-aza-deoxycytidine and TSA treatments.

\begin{tabular}{|c|c|c|c|c|c|c|}
\hline & \multicolumn{2}{|c|}{ PTCH } & \multicolumn{2}{|c|}{ SMO } & \multicolumn{2}{|c|}{ GLI3 } \\
\hline & Before 5'-aza & After 5'-aza & Before 5'-aza & After 5'-aza & Before 5'-aza & After 5'-aza \\
\hline \multicolumn{7}{|l|}{ MB cell lines } \\
\hline TE671 & ++ & ++ & ++ & ++ & ++++ & ++++ \\
\hline PFSK-1 & + & ++ & ++ & +++ & +++ & +++ \\
\hline DAOY & + & + & ++ & ++ & +++ & +++ \\
\hline TE671c2 & ++ & +++ & ++ & +++ & ++++ & ++++ \\
\hline D283 & + & ++ & + & ++ & ++ & ++ \\
\hline SK-PN-DW & + & ++ & ++ & +++ & + & + \\
\hline \multicolumn{7}{|l|}{ GB cell lines } \\
\hline U87MG & $+/-$ & + & $+/-$ & ++ & ++ & ++ \\
\hline A172 & + & ++ & + & + & ++ & ++ \\
\hline LN405 & + & ++ & ++ & ++ & +++ & +++ \\
\hline SW1783 & $+/-$ & ++ & + & + & ++ & ++ \\
\hline T98G & + & + & + & + & ++ & ++ \\
\hline SW1088 & ++ & ++ & + & + & ++ & ++ \\
\hline CCF-STTG & + & + & ++ & ++ & ++ & ++ \\
\hline GOS-3 & + & + & + & + & ++ & ++ \\
\hline \multicolumn{7}{|l|}{ NB cell lines } \\
\hline SK-N-F1 & + & ++ & $+/-$ & + & - & + \\
\hline SK-N-Be(2) & + & + & + & + & - & + \\
\hline SK-N-MC & + & ++ & ++ & ++ & ++ & ++ \\
\hline KELLY & + & ++ & + & + & - & - \\
\hline MC-IXC & + & ++ & ++ & ++ & ++ & ++ \\
\hline SH-SY-5Y & ++ & ++ & ++ & ++ & - & + \\
\hline MHH-MB-11 & + & ++ & ++ & ++ & - & - \\
\hline SK-N-DZ & + & + & + & + & ++ & ++ \\
\hline IMR-32 & + & + & + & + & + & + \\
\hline SIMA & ++ & ++ & ++ & ++ & - & + \\
\hline $\mathrm{Be}(2) \mathrm{C}$ & + & + & + & ++ & - & + \\
\hline
\end{tabular}

MB, medulloblastoma; GB, glioblastoma; NB, neuroblastoma; -, not expressed; +/-, not clear level of expression; + to ++++, different levels of expression.

deoxycytidine and TSA treatments (Fig. 3). Also, the glioblastoma cell line U87MG and two neuroblastoma cell lines [SK-N-F1 and $\mathrm{Be}(2) \mathrm{c}]$ presented re-expression of the gene after the treatments (Fig. 3). The melting curves supported a partial/ hemimethylation status at the SMO promoter (Fig. 4). In order to corroborate this result in tumor samples, 40 glioblastomas and 40 neuroblastomas were studied by qMSP and melting curve analysis (Fig. 4). Of these, 24 glioblastomas $(60 \%)$ and 16 neuroblastomas $(40 \%)$ presented methylation or partial/hemimethylation of the SMO gene promoter region.

\section{Discussion}

In this study we checked the role of the Shh pathway in the development of two highly malignant brain tumors (glio- blastoma and medulloblastoma) and in neuroblastoma, a malignant extracranial tumor of the perypheral nervous system. In fact we incorporated medulloblastoma as a control, as its participation in the Shh pathway is well known (14-16). These three tumors correspond to the most malignant phenotypes of tumors of the nervous system in adults (glioblastoma) and children (medulloblastoma and neuroblastoma). The three tumors present different genetic profiles. From the histopathological point of view, there is a connection between medulloblastoma and neuroblastoma in the sense that both of them belong to the so-called small round cell tumors. Medulloblastomas are considered to be primitive neuroectodermal tumors (PNET) developed in the cerebellum. Furthermore, certain types of supratentorial PNET, are diagnosed as brain neuroblastomas when they present neuroblastic differentiation. 


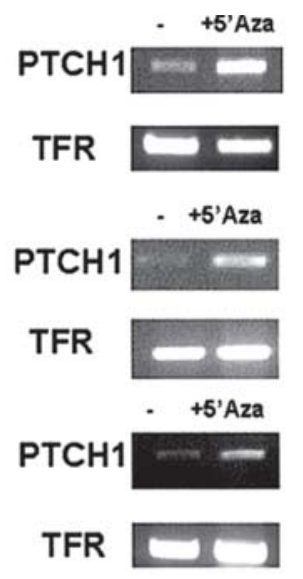

A

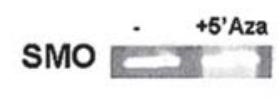

TFR
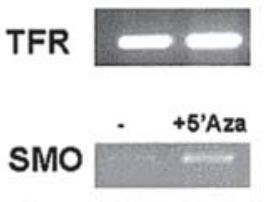

TFR

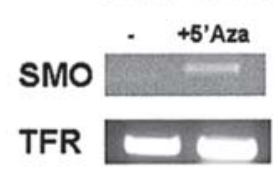

$\mathrm{Be}(2) \mathrm{C}$

SW1783

ETCH

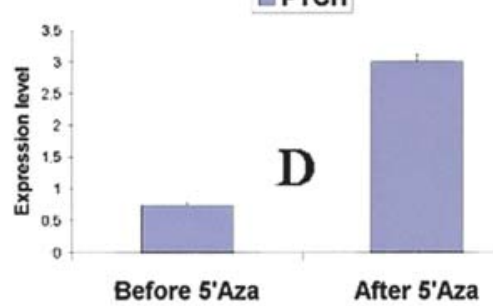

B
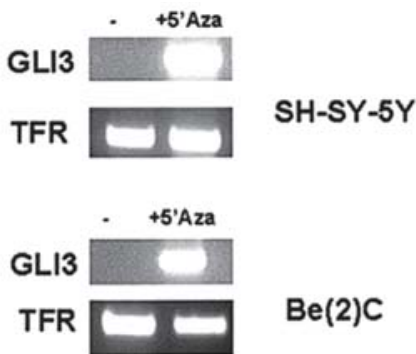

$\mathrm{Be}(2) \mathrm{C}$

C

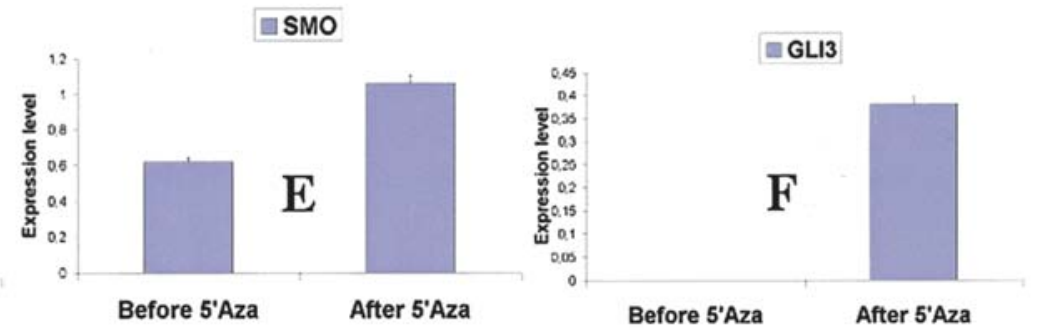

Figure 3. Comparative RNA expression of PTCH (A and D), SMO (B and E) and GLI3 (C and F) before and after treatments with 5-aza-2'-deoxycytidine and TSA, determined by standard (A-C) and quantitative (D-F) RT-PCR. TFR was included as a control of RNA expression. p-values, 0.09736 (SMO); 0.001023 (PTCH); and $0.01884(G L I 3)$.
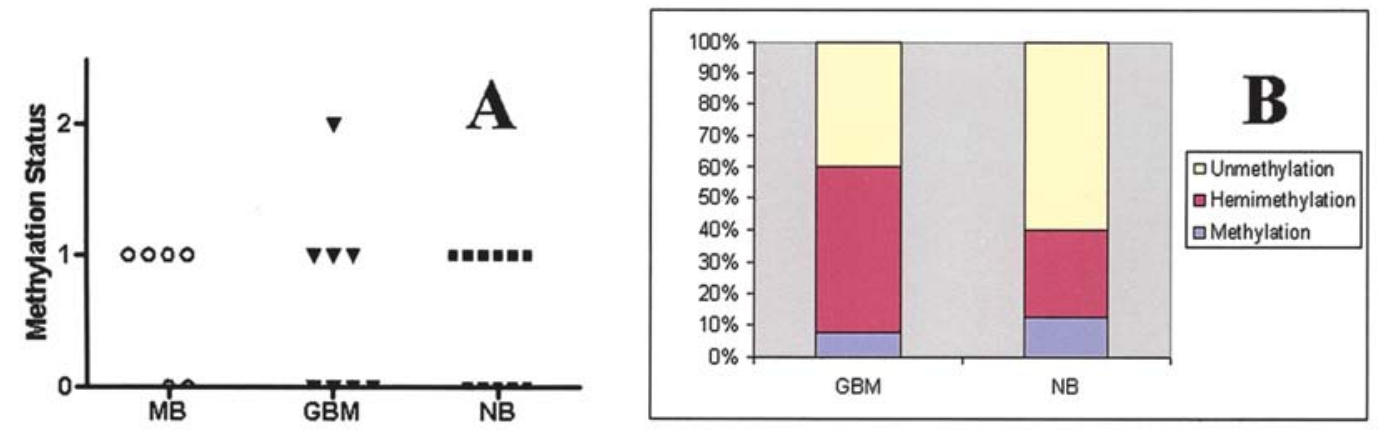

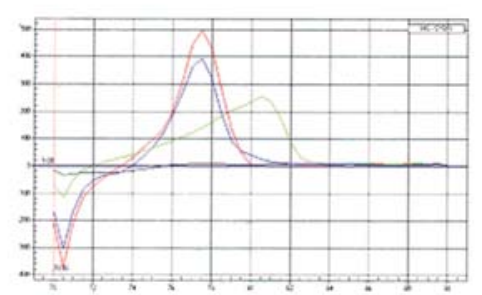

C

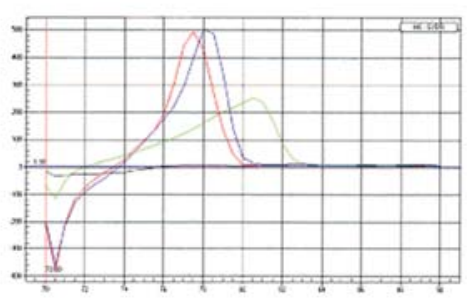

D

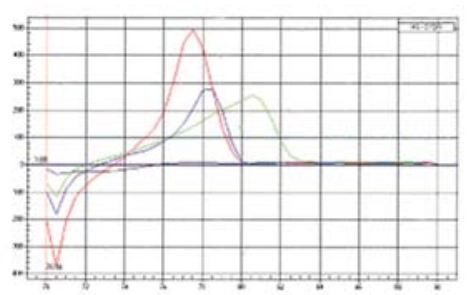

$\mathbf{E}$

Figure 4. SMO promoter methylation status in cell lines (A) and tumor samples (B) determined by qRT-PCR. Melting curves correspond to unmethylated (C), partial/hemimethylated (D), and completely methylated (E) SMO promoter in three different cell lines. Melting curves refer to blood DNA as a negative control (red), universal methylated DNA (Chemicon International) as a positive control (green), DNA tumor sample (blue), and non-template control (black). For interpretation, we referred to partial/hemimethylation when the melting temperature of the sample was $0.5-1^{\circ} \mathrm{C}$ higher than the melting temperature of the normal control, while a higher difference would correspond to complete methylation. MB, medulloblastoma; GBM, glioblastoma; NB, neuroblastoma. 
In this sense, in studies on medulloblastoma, neuroblastoma and supratentorial PNET it is worthwhile to define specific genetic markers that allow a more accurate diagnosis and a more specific treatment of these relatively similar lessions. Embryologically speaking, glioblastoma and medulloblastoma derive from the neuroepithelium, while neuroblastoma from the neural crest, as other tumors that appear outside of the nervous system (melanoma, thyroid medullary carcinoma, small cell lung cancer, malignant schwannoma, among others).

The Shh pathway is an evolutionarily conserved developmental cassette, leading different tasks in the developing organism, such as the development of the neural tube, craniofacial structures, limb, lung, and others, to ensure proper positional or polarity information within part of the developing embryo. The activation of the Shh pathway is conducted through Shh, which triggers a derepression of the negative modulation of the pathway executed by Ptch on Smo. The net effect of Shh binding to the cell surface complex is an activation of the pathway by downstream activators such as Gli1.

We have checked the expression of some of the major Shh pathway genes (PTCH, SMO, GLII and GLI3) in medulloblastoma, neuroblastoma and glioblastoma cell lines, together with glioblastoma and neuroblastoma samples. Methylation studies were applied on SMO as well.

All medulloblastoma cell lines (except DAOY) and all glioblastoma cell lines (except GOS-3) showed higher levels of expression of GLII than in normal adult brain RNA expression. However, only 3 of the 11 neuroblastoma cell lines (SK-N-MC, MC-IXC and SH-SY-5Y) showed higher expression of GLI1 than in normal adult brain RNA. This high expression level of GLII supports the Shh pathway activation (17) in glioblastoma and medulloblastoma, and, less frequently, in neuroblastoma. On the contrary, 2 medulloblastoma cell lines (DAOY, SK-PN-DW), 7 out of 8 cell lines of glioblastoma (all except SW1088), half of the glioblastoma samples (12 of 25) and 4 neuroblastoma cell lines [SK-N-MC, KELLY, MC-IXC and Be(2)C] showed lower PTCH expression than in normal adult brain RNA.

We detected SMO promoter methylation in 4 medulloblastoma cell lines (PFSK-1, TE671c2, D283 and SK-PNDW), 4 glioblastoma cell lines (U87MG, A172, LN405 and SW1088), and 6 neuroblastoma cell lines [SK-N-FI, SK-NMC, MHH-NB-11, SK-N-DZ, SIMA and Be(2)c]. The partial/ hemimethylation observed was also supported by the melting curves obtained from glioblastoma and neuroblastoma tumor samples.

Expression of GLI3 in all medulloblastoma and glioblastoma cell lines and in almost all glioblastoma samples (except two samples) was higher than in normal adult brain RNA. However, similar results were obtained in only 4 out of 11 neuroblastoma cell lines (SK-N-MC, MC-IXC, SK-N-DZ and IMR-32).

We have demonstrated that the Shh pathway is activated in medulloblastoma, glioblastoma and neuroblastoma. Previously, the activation of this pathway in medulloblastoma was known (14), but, it is not yet fully understood whether glioblastoma $(18,19)$ and neuroblastoma are involved in the activation of the Shh pathway.
It was reported that high expression of $P T C H$ implies the Shh pathway activation in tumors of the digestive tract (17). In our study $P T C H$ expression was lower than in normal controls, but $P T C H$ re-expression after treatments with 5-aza-2'deoxycytidine and TSA supports its promoter methylation, as demonstrated for breast cancer (20).

In this study, we paid special attention to the relationship between the expresion of $S M O$ and $G L I 3$, as they act together, once the Shh pathway has been initiated, to make the expression of GLII possible. Once this is initiated, a cascade of genes taking part in apoptosis control and regulation of growth will be activated. Smo behaves as an oncogene, as its activation by Shh promotes Gli1 activation. On the contrary, Gli3 exerts a dual possibility: as an activator (similarly to Gli1 and Gli2), or as a repressor (21) that brakes the activating function of Smo on Gli1.

We demonstrated that SMO acts as an activator of the Shh pathway in medulloblastoma, glioblastoma and neuroblastoma, as we detected a good level of expresion of SMO in most cell lines and tumor samples studied. We also demonstrated the methylation of $S M O$ promoter in cell lines and tumors, and the fact that expression of SMO increases after 5-aza-2'deoxycytidine and TSA treatments in cell lines. Nevertheless it seems methylation is not playing a significant role in silencing this gene.

Medulloblastoma and glioblastoma cell lines showed a very noticeble correlation between SMO and GLI3 RT-PCR expression, with the exception of the U87MG glioblastoma cell line, which did not show a clear expression level of SMO, although after treating the cell line with 5-aza-2'-deoxycytidine and TSA, re-expresion of SMO was observed. These results were confirmed by qRT-PCR analysis before and after treatments with demethylating agents, and by qMSP that revealed melting curves compatible with partial/hemimethylation of SMO in the U87MG cell line.

Contrary to medulloblastoma and glioblastoma, neuroblastoma cell lines did not show a clear correlation between $S M O$ and GLI3 expression. Seven of $11(64 \%)$ cell lines presented $S M O$ expression but did not express GLI3. Two of these 7 cell lines did not even express GLI3 after treatments with 5-aza-2'deoxycytidine and TSA, while the other 5 did. The rest of the cell lines (4 of 11,36\%) showed SMO and GLI3 expression. qMSP also supported $S M O$ promoter partial/hemimethylation in neuroblastoma cell lines and tumor samples.

After the analysis of SMO and GLI3 expression we concluded that $S M O$ gene expression seems to be required for GLI3 expression in medulloblastoma and glioblastoma, as shown by the clear correlation of expression of both genes in these types of brain tumors, and that Gli3 is acting as an activator of the Shh pathway in these tumors. On the contrary, neuroblastomas present different results, as the correlation between SMO and GLI3 expression is seen in only 4 of 11 cell lines. In these cases, we would equally suggest Gli3 as an activator of Shh. But the other 7 cell lines present a different scenario: 5 of them re-express GLI3 after the treatments with 5-aza-2'-deoxycytidine and TSA, which even suggests, as commented for PTCH, that GLI3 might be subjected to promoter methylation to epigenetically regulate its own expression, independently of Shh. The fact that in the other 2 cell lines no expression is produced even after the 
treatments with 5-aza-2'-deoxycytidine and TSA indicates a non-activator but a repressor function for Gli3 in at least a subset of neuroblastomas. Also a gradient-like expression of other genes, SMO included e.g., might be needed for GLI3 expression, in an Shh-dependent or -independent manner.

The fact that a clear activation of the Shh pathway has been demonstrated by our study in glioblastoma and medulloblastoma, but not clearly enough in neuroblastoma, suggests different embryological ethiology of these tumors, as they are neuroepithelial in origin, while neuroblastoma derives from the neural crest. This embryological difference might in part explain the differences in the involvement of the Shh demonstrated in our study for these three malignant tumors of the nervous sytem. Further studies are required to evaluate our hypotheses.

\section{Acknowledgements}

M.H. Shahi is a fellow of AECI (Agencia Española de Cooperación Internacional), Madrid, Spain. Aiala Lorente received a predoctoral fellowship from the Department of Education, Universities and Research of the Basque Government, Vitoria, Spain. We thank Paula Lázcoz for general instruction on cell culture, and Xing Fan for help with the GraphPad software. We are grateful to the Chairman of the Department of Zoology at Aligarh Muslim University, Aligarh, India, for allowing M.H. Shahi to perform a part of his PhD experiments at the University of Navarra, in Pamplona, Spain. J.S. Castresana thanks the Asociación Española de Pediatría, Madrid, for the VIII Premio Nutribén de Investigación Pediátrica. This research was supported in part by grants from the Departamento de Salud del Gobierno de Navarra, Pamplona, Fondo de Investigación Sanitaria (PI031356), Ministerio de Ciencia y Tecnología y Fondo Europeo de Desarrollo Regional (BFI2003-08775), and Fundación Mapfre Medicina, Madrid.

\section{References}

1. Kimura H, Stephen D, Joyner A and Curran T: Gli1 is important for medulloblastoma formation in Ptc1+/- mice. Oncogene 24: 4026-4036, 2005.

2. Gorlin RJ: Multiple myeloma. Gerodontics 1: 249-251, 1985.

3. Stone DM, Hynes M, Armanini M, et al: The tumour-suppressor gene patched encodes a candidate receptor for Sonic hedgehog. Nature 384: 129-134, 1996.

4. Kenney AM and Rowitch DH: Sonic hedgehog promotes G(1) cyclin expression and sustained cell cycle progression in mammalian neuronal precursors. Mol Cell Biol 20: 9055-9067, 2000 .
5. Micchelli CA, The I, Selva E, Mogila V and Perrimon N: Rasp, a putative transmembrane acyltransferase, is required for Hedgehog signaling. Development 129: 843-851, 2002.

6. Kinzler KW, Bigner SH, Bigner DD, et al: Identification of an amplified, highly expressed gene in a human glioma. Science 236 : 70-73, 1987 .

7. Cohen MM Jr: Perspectives on overgrowth syndromes. Am J Med Genet 79: 234-237, 1998.

8. Ingham PW and McMahon AP: Hedgehog signaling in animal development: paradigms and principles. Genes Dev 15: 3059-3087, 2001.

9. Wang B, Fallon JF and Beachy PA: Hedgehog-regulated processing of Gli3 produces an anterior/posterior repressor gradient in the developing vertebrate limb. Cell 100: 423-434, 2000 .

10. Dahmane N, Lee J, Robins P, Heller P and Ruiz i Altaba A: Activation of the transcription factor Gli1 and the Sonic hedgehog signalling pathway in skin tumours. Nature 389: 876-881, 1997.

11. Katoh Y and Katoh M: Identification and characterization of rat Desert hedgehog and Indian hedgehog genes in silico. Int J Oncol 26: 545-549, 2005.

12. Zhu Y, James RM, Peter A, Lomas C, Cheung F, Harrison DJ and Bader SA: Functional Smoothened is required for expression of GLI3 in colorectal carcinoma cells. Cancer Lett 207: 205-214, 2004.

13. Herman JG, Graff JR, Myohanen S, Nelkin BD and Baylin SB: Methylation-specific PCR: a novel PCR assay for methylation status of CpG islands. Proc Natl Acad Sci USA 93: 9821-9826, 1996.

14. Berman DM, Karhadkar SS, Hallahan AR, et al: Medulloblastoma growth inhibition by hedgehog pathway blockade. Science 297: 1559-1561, 2002.

15. Di Marcotullio L, Ferretti E, De Smaele E, et al: REN(KCTD11) is a suppressor of Hedgehog signaling and is deleted in human medulloblastoma. Proc Natl Acad Sci USA 101: 10833-10838, 2004.

16. Piedimonte LR, Wailes IK and Weiner HL: Medulloblastoma: mouse models and novel targeted therapies based on the Sonic hedgehog pathway. Neurosurg Focus 19: E8, 2005.

17. Berman DM, Karhadkar SS, Maitra A, et al: Widespread requirement for Hedgehog ligand stimulation in growth of digestive tract tumours. Nature 425: 846-851, 2003.

18. Clement V, Sanchez P, De Tribolet N, Radovanovic I and Ruiz i Altaba A: HEDGEHOG-GLI1 signaling regulates human glioma growth, cancer stem cell self-renewal, and tumorigenicity. Curr Biol 17: 165-172, 2007.

19. Ehtesham M, Sarangi A, Valadez JG, et al: Ligand-dependent activation of the hedgehog pathway in glioma progenitor cells. Oncogene 26: 5752-5761, 2007.

20. Wolf I, Bose S, Desmond JC, Lin BT, Williamson EA, Karlan BY and Koeffler HP: Unmasking of epigenetically silenced genes reveals DNA promoter methylation and reduced expression of PTCH in breast cancer. Breast Cancer Res Treat 105: 139-155, 2007.

21. Meyer NP and Roelink H: The amino-terminal region of Gli3 antagonizes the Shh response and acts in dorsoventral fate specification in the developing spinal cord. Dev Biol 257: 343-355, 2003. 